\title{
Photonic Generation of Triangular Shaped Microwave Pulses Using Stimulated Brillouin Scattering (SBS)
}

\author{
M.Baskaran ${ }^{1}$, S.Sevagan ${ }^{2}$, K.Vijayakumar ${ }^{3}$ \\ \{ baski.maha@gmail.com ${ }^{1}$, sevaindia.sts@gmail.com ${ }^{2}$, mkvijay@msn.com ${ }^{3}$ \} \\ Department of Electronics and Communication Engineering, Sri Sairam Engineering College, \\ Chennai-600044, India ${ }^{1}$, Department of Electronics and Communication Engineering, \\ Sri Venkateswaraa College of Technology, Chennai-602105, India ${ }^{2}$, Department of Computer Science \\ and Engineering, St.Joseph's Institute of Technology, Chennai-600119, India ${ }^{3}$.
}

\begin{abstract}
A triangular shaped microwave pulse is generated by optical heterodyning principle using Stimulated Brillouin Scattering (SBS) in an optical fiber communication link. A narrowband Distributed Feedback (DFB) Laser diode of wavelength $1552.52 \mathrm{~nm}$, LiNbO Mach Zehnder Modulator (MZM), Highly Nonlinear Fiber (HNLF) of length 20 $\mathrm{Km}$ and Optical receiver that comprise high power optical pump source, circulator, PIN photo detector and Band pass filter form the link. Finally, a Radio over Fiber system for the generation of triangular shaped microwave pulse is built through simulation.
\end{abstract}

Keywords: Stimulated Brillouin Scattering (SBS), Triangular Shaped Pulses, DFB Laser, External Modulator.

\section{Introduction}

Microwave pulse generation utilizing effectively accessible electrical methodologies and devices used for generation have the limitation of low frequency and therefore the bandwidth capacity is less. Luckily, the inherent benefit of photonics makes the generation of microwave competent of providing huge bandwidth (e.g., 10's to 100's of GHz). The triangular shaped waveform is the standard one amongst a wide variety of microwave pulses, which can be generated using different optical approaches. The fundamental ideas of these methods are derived from the study of periodic signals by Fourier series. As triangular shaped waveform comprises only odd-order harmonics and has a quick roll-off in the amplitude of its higher order harmonics and approximately, two odd-order harmonics will roughly reflect the triangular waveform. One solution is to construct a triangular shaped pulse generator in association with the frequency to time mapping using the spectral shaping. However, it could lead to bad control of the generated signal parameters. Furthermore, high environmental reliability and proper phase synchronization are necessary due to the utilization of cascaded optical filters. It is possible to achieve an amplitude proportion of $1: 9$ and a frequency proportion of 1:3 through changing the amplitudes and phases of the sidebands for the two harmonics that is equal to the first two triangular waveform components of the Fourier series. Hence, the optical approach supported external optical modulation is suggested to give solution to the above issues. In the suggested solution, only optical sidebands of odd-order are produced using an MZM biased at minimal transmission point. As an outcome of this approach, an optical single-drive Mach-Zehnder interferometer modulator in combination 
with a polarization modulator or an interleaver in a Sagnac Loop could produce triangularshaped microwave pulses.

In addition, a dual-parallel MZM wherein six dc biases can also produce triangular shaped microwave pulses and to get specified harmonics, it must be controlled precisely and simultaneously. Owing to the utilization of Sagnac Loop configuration or Mach-Zehnder interferometer, these two devices may be susceptible to environmental changes. The firstorder sideband amplitude is 9 times that of the third-order sideband by varying the modulation index accordingly. Therefore to contribute gain for retrieving suppressed carrier, the SBS based optical carrier processing is implemented. For eliminating the unnecessary sidebands, an optical band pass filter is then employed. Thus triangular-shaped microwave pulses are often produced in this manner when SBS gain is sufficiently large. As a result, a reliable generation device with good precision is therefore assured.

For stimulated Brillouin scattering based radio over-fiber (RoF) systems, a harmonic radio frequency $(\mathrm{RF})$ carrier generation and the up conversion method for broadband data with single mode and SSB modulation has been suggested. Through this technique, we have successfully exhibit $3^{\text {rd }}$-harmonic radio frequency carrier generation at frequency $32.625 \mathrm{GHz}$ with local oscillator frequency of $10.875 \mathrm{GHz}$ and up conversion of $1.25 \mathrm{Gbps}$ data to the RF carrier. Furthermore, the data capacity is independent of the SBS gain profile. At last, the downlink RoF system's transmission performance is evaluated [1]. A triangular shaped pulse generation by means of optical carrier processing based on SBS has been experimentally demonstrated. In that, by changing the RF signal frequency supplied to the MZM, the triangular pulse has been produced and its frequency is often tuned flexibly. And in the experiments, triangular shaped pulses are generated with frequencies $5 \mathrm{GHz}, 8 \mathrm{GHz}$ and 10 $\mathrm{GHz}$ according to the above theory, demonstrating desired waveforms and excellent tunability [2]. Stimulated Brillouin Scattering allows the pump light to spread backwards and this successively causes the distributed erbium doped fiber amplifier (DEDFA) gain to decrease. This paper provides a study of Stimulated Brillouin Scattering nonlinear effect on DEDFA and presents descriptive numerical findings. And it is demonstrated that there exists a pump laser linewidth threshold value. The DEDFA gain is decreased substantially at the point when the linewidth pump laser is smaller than the threshold value [3].

An extremely steady configuration of optical two-tone signal generator intended for astronomical implementations and high data rate communication produces high frequency (HF) optical signals (frequencies over $100 \mathrm{GHz}$ ). One way of generating these optical signals is to produce by means of two laser sources with an optical phase locked loop (PLL) for feedback control; on the other hand, the optical PLL has a stability issues with its operation [4]. The optical intensity and its mathematical formulae relating to the Fourier expansion of the standard triangular shaped waveform are often produced by the use of active polarization control and optical carrier suppression modulation. The repetition rate of the pulses is also observed to depend only on the local oscillator driving frequency. Hence, continuously the frequency tunability is achieved by adjusting it. First the function is evaluated by theory and then tested by simulation. As tuning the repetition rate involves just one frequency tunable oscillator, the proposal may be appealing [5]. The simulation investigated the optical carrier suppressed signal generation and performances of radio over fiber system. A Dual-Drive Mach-Zehnder Interferometer is engaged for both signal modulation and suppression of optical carrier. By biasing the Dual-Drive MZM at null point, Suppressed carrier modulation 
is accomplished. Suppression of optical carriers increases efficiency of RoF system like noise figure and link gain. And Different optical carrier suppression ratio values are often achieved by changing the Dual-Drive MZM extinction ratio [6]. The paper presented an all-optical millimetre wave carrier generation from a multi wavelength Brillouin erbium fiber laser. By controlling the gain in the cavity, Four-channel outputs are generated from the fiber laser, with spacing of about $21.5 \mathrm{GHz}$. As a result of eliminating the two channels in the centre, the dual wavelength signal with spacing corresponds to 6 orders of the Brillouin frequency shift is achieved. A millimetre wave carrier at frequency $64.17 \mathrm{GHz}$ is produced by heterodyning these signals at the speedy photo detector. The versatility of the induced millimetre wave signal frequency to be tuned at $0.0066 \mathrm{GHz} /{ }^{\circ} \mathrm{C}$ is realised by the Brillouin frequency shift characteristic of temperature dependence [7].

\section{Principle}

The principle of optical MMW generation is shown in fig. 1. Initially the optical signal from a DFB laser source is given to the MZM (Mach-Zehnder Modulator). An RF sinusoidal signal is also fed into the MZM modulator. In the MZM modulation index is set in such a way that the optical carrier and even order side and are suppressed at its output. Hence, the optical signal at the MZM modulator output contains odd order side bands only $(1,3,5, .$.$) , whereas the$ even order side bands $(2,4,6, .$.$) and carrier are suppressed. The side bands can be increased by$ increasing the extension ratio of the MZM modulator. But optical carrier is necessary for generation of triangular waves.

$$
E_{\text {out }}=E \exp \left(j \omega_{c} t\right) \sum_{n=-\infty}^{\infty} \frac{1-(-1)^{n}}{2} J_{n} \exp (j n \omega t)
$$

We can consider upto $\pm 3^{\text {rd }}$ side band,

$$
\begin{gathered}
E_{\text {out }}=E_{0} \exp \left(j \omega_{\mathrm{c}} \mathrm{t}\right) \times \frac{1-(-1)^{-3}}{2} J_{-3} \exp (-j 3 \omega t)+\frac{1-(-1)^{-2}}{2} J_{-2} \exp (-j 2 \omega t)+ \\
\frac{1-(-1)^{-1}}{2} J_{-1} \exp (-j \omega t)+\frac{1-(-1)^{0}}{2} J_{0} \exp (-j 0 \omega t)+ \\
\frac{1-(-1)^{1}}{2} J_{1} \exp (j \omega t)+\frac{1-(-1)^{2}}{2} J_{2} \exp (j 2 \omega t)+ \\
\left.\frac{1-(-1)^{3}}{2} J_{3} \exp (j 3 \omega t)\right\} \\
E_{\text {out }}=E_{o} \exp \left(j \omega_{\mathrm{c}} \mathrm{t}\right) \times\left\{\begin{array}{c}
J_{-3} \exp (-j 3 \omega t)+0+J_{-1} \exp (-j \omega t)+0+J_{1} \exp (j \omega t)+ \\
\left.0+J_{3} \exp (j 3 \omega t)\right\}
\end{array}\right. \\
E_{\text {out }}=E_{o} \exp \left(j \omega_{\mathrm{c}} \mathrm{t}\right) \times\left\{J_{-3} \exp (-j 3 \omega t)+J_{-1} \exp (-j \omega t)+J_{1} \exp (j \omega t)+\right. \\
\left.J_{3} \exp (j 3 \omega t)\right\}
\end{gathered}
$$

The expression (2) contains the optical carrier and odd order sidebands only and even order side bands are suppressed. But practically they cannot be completely suppressed. Hence the expression (1) can be rewritten as, 


$$
\begin{array}{r}
E_{\text {out }}=E_{o} \exp \left(j \omega_{c} t\right) \times\left\{\sqrt{\alpha} \sum_{n=-\infty}^{\infty} J_{2 n} \exp [j(2 n) \omega t]+\right. \\
\left.\sum_{n=-\infty}^{\infty} J_{2 n+1} \exp [j(2 n+1) \omega t]\right\}
\end{array}
$$

Where, ' $\alpha$ '- the power coefficient of the vestigial even-order sidebands and the carrier.

The carrier suppression ratio (CSR) is given by

$$
\begin{aligned}
\mathrm{CSR} & =10 \log _{10}\left(\mathrm{P}_{1 \text { st-order }} / \mathrm{P}_{\text {carrier }}\right) \\
& =20 \log _{10}\left(\mathrm{~J}_{1} / \sqrt{\alpha} \mathrm{J}_{0}\right)
\end{aligned}
$$

Where, $\mathrm{P}_{\text {carrier }}$ and $\mathrm{P}_{1 \text { st- order }}$ are the powers of optical carrier and first order sideband respectively. Hence

$$
\alpha=\frac{\mathrm{J}_{1}{ }^{2}}{\mathrm{~J}_{0}{ }^{2}\left(10^{\operatorname{CSR} / 10}\right)}
$$

So as to recover the suppressed carrier, SBS technique is used. SBS involves the use of a laser source followed by EDFA (pumping source) and a Bidirectional HNLF (Highly NonLinear Fiber). The MZM output is connected to source side of the fiber through an isolator. The pumping signal is applied to the fiber through a circulator. When the laser power exceeds a particular threshold level of the fiber, non-linearity occurs in the fiber. The vestigial carrier would then be amplified with the SBS gain of G. In the meantime, an optical band pass filter is employed to filter the unnecessary sidebands, since the optical field can be written at the output of the OBPF as,

$$
\begin{gathered}
\mathrm{E}_{\text {out }}=\mathrm{E}_{\mathrm{O}} \exp \left(\mathrm{j} \omega_{\mathrm{c}} \mathrm{t}\right) \times\left\{\sqrt{\mathrm{G} \alpha} \mathrm{J}_{0} \exp (\mathrm{j} \phi)+\mathrm{J}_{1} \exp (\mathrm{j} \omega \mathrm{t})+\right. \\
\left.\sqrt{\alpha} \mathrm{J}_{2} \exp (\mathrm{j} 2 \omega \mathrm{t})+\mathrm{J}_{3} \exp (\mathrm{j} 3 \omega \mathrm{t})\right\} \\
\mathrm{I}_{\text {out }} \approx 2 \mathrm{RE}_{0}{ }^{2}\left\{\sqrt{\mathrm{G} \alpha} \mathrm{J}_{0}\left[\mathrm{~J}_{1} \cos (\omega \mathrm{t}-\phi)+\mathrm{J}_{3} \cos (3 \omega \mathrm{t}-\phi)\right]+\mathrm{DC}+\mathrm{J}_{1} \mathrm{~J}_{3} \cos (2 \omega \mathrm{t})\right\}
\end{gathered}
$$

From Equation (7), it is obvious that, the Second order harmonic is the most important part, that stimulates noise to the triangular shaped pulse. Thus the following condition must be satisfied in Equation (7) that

$$
\sqrt{\mathrm{G} \alpha} \mathrm{J}_{0} \mathrm{~J}_{3} \gg \mathrm{J}_{1} \mathrm{~J}_{3}
$$

The Second order harmonic are often eliminated such that the system should have sufficiently large SBS gain. While setting the index of modulation to 1.51 , it is possible to further simplify Equation (7) to,

$$
\mathrm{I}_{\text {out }} \alpha \frac{\mathrm{DC}}{\sqrt{\mathrm{G} \alpha} \mathrm{J}_{0}}+\cos (\omega \mathrm{t}-\phi)+\frac{1}{9} \cos (3 \omega \mathrm{t}-\phi)
$$


When the SBS gain ' $G$ ' is at peak, the nonlinear phase change induced by SBS to be zero. (i.e.) $\varphi=0$. Mathematical expression has been obtained approximately in Equation (9) to describe triangular microwave pulse.

$$
\mathrm{I}_{\text {out }} \alpha \frac{\mathrm{DC}}{\sqrt{\mathrm{G} \alpha} \mathrm{J}_{0}}+\cos (\omega \mathrm{t})+\frac{1}{9} \cos (3 \omega \mathrm{t})
$$

Due to this non-linearity, most of the pumping signal is reflected back to the source side of the fiber. Hence this pumping signal is superimposed on the suppressed carrier and the carrier signal is recovered. An OBPF (Optical Band pass Filter) is used to filter out the side bands with negative order. Hence, its output contains only positive order side bands $(+1,+2,+5, \ldots)$ and all negative order side bands $(-1,-3,-5, \ldots)$ are removed. This filtered optical signal is then given to a PIN photo detector.

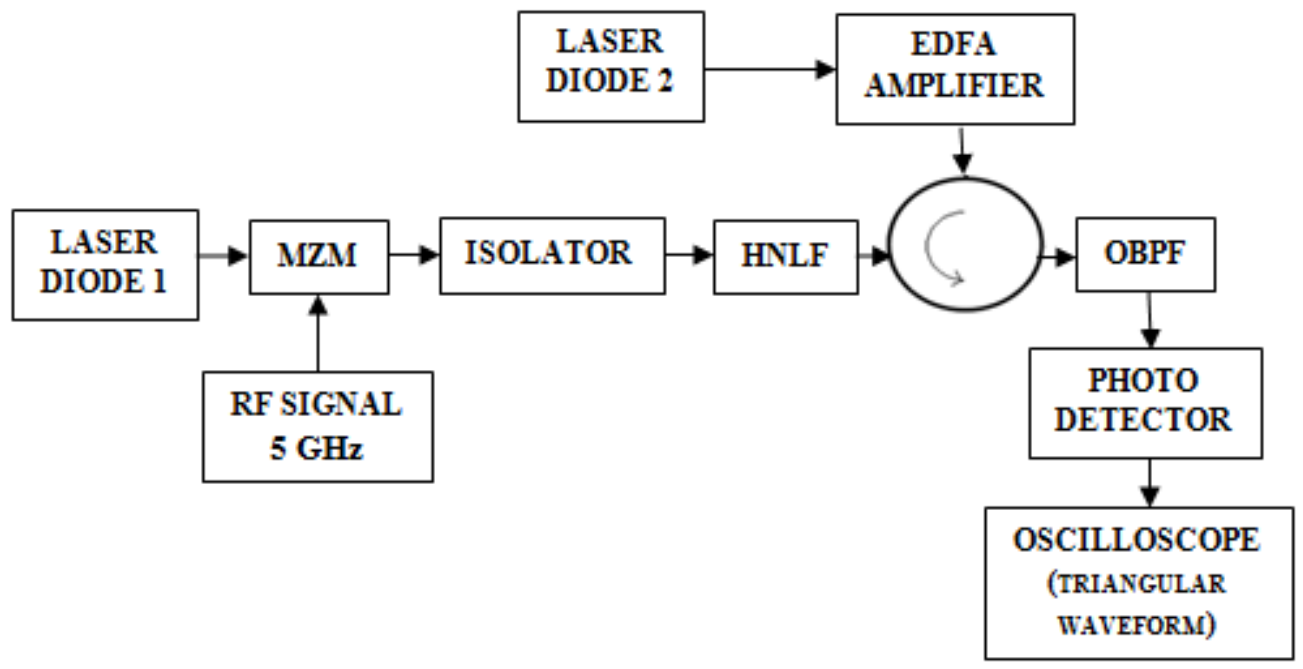

Fig. 1. Simulation setup for the generation of triangular shaped microwave pulse using SBS.

At the PIN photo detector output, we can get the required triangular waveform. The frequency of the triangular waveform generated is often altered by varying the input sinusoidal (RF) signal frequency.

\section{Simulation Results}

The simulation has been carried out with the aid of Optisystem software. The input spectrum obtained from the laser diode is shown in Fig. 2. The frequency of $193.1 \mathrm{THz}$ signal having the wavelength of $1550 \mathrm{~nm}$ is used as the optical input source. This input signal is applied to Mach-Zehnder Modulator for modulation using a RF signal. 


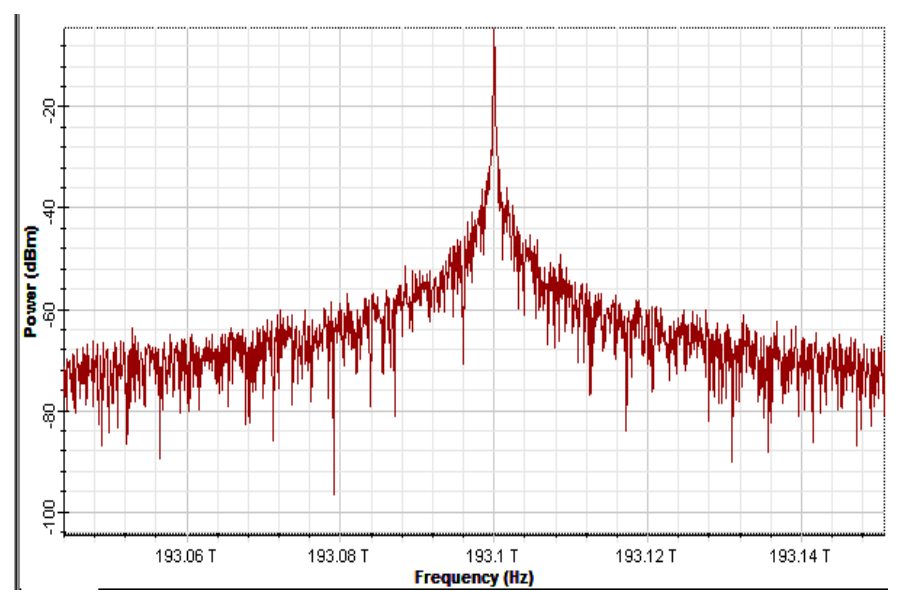

Fig. 2. Spectrum of Laser diode.

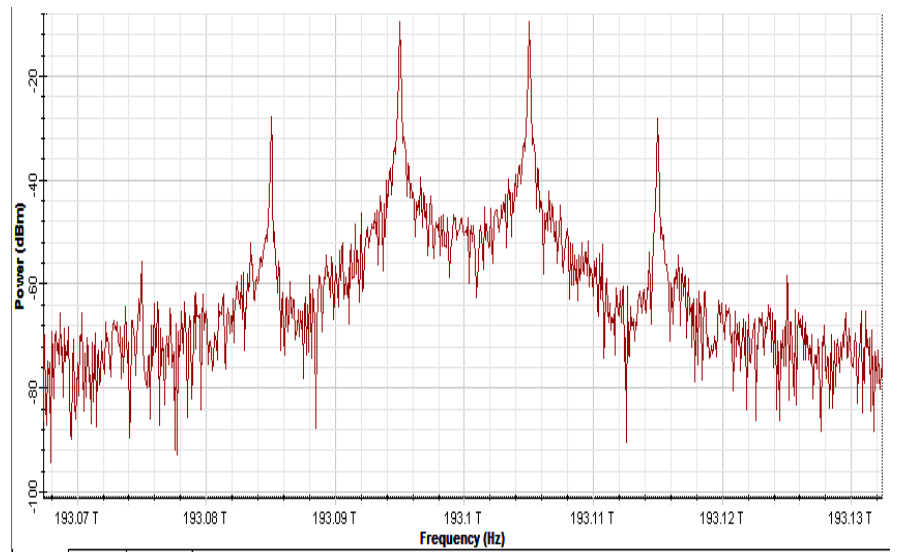

Fig. 3. Output Spectrum of MZM

The suppressed carrier and suppressed even order side bands from MZM is shown in Fig. 3. The applied input signal is modulated using Mach-Zehnder modulator. From the output of MZM modulator we obtain only Odd order side bands. Because of the constructive and destructive interference of the optical signal produced by Lithium Niobate Crystal in MZM we get only odd order side bands.

At the receiver, The RF output obtained from the photo detector is shown in Fig. 4. The photo detector converted the optical signal as electrical signal. Then the generated RF signal contains the carrier and odd order sidebands only. The second order sidebands are often completely suppressed by appropriately increasing the carrier signal power. 


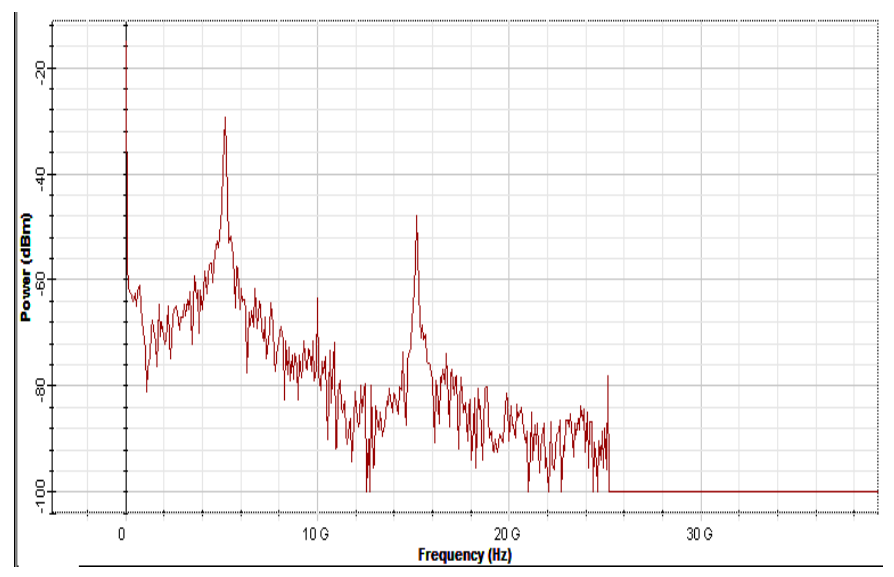

Fig. 4. RF output spectrum

The generated triangular shaped pulse is shown in Fig. 5. The triangular waveforms of different frequencies can be achieved by fine-tuning the RF signal frequency supplied to the Mach-Zehnder modulator.

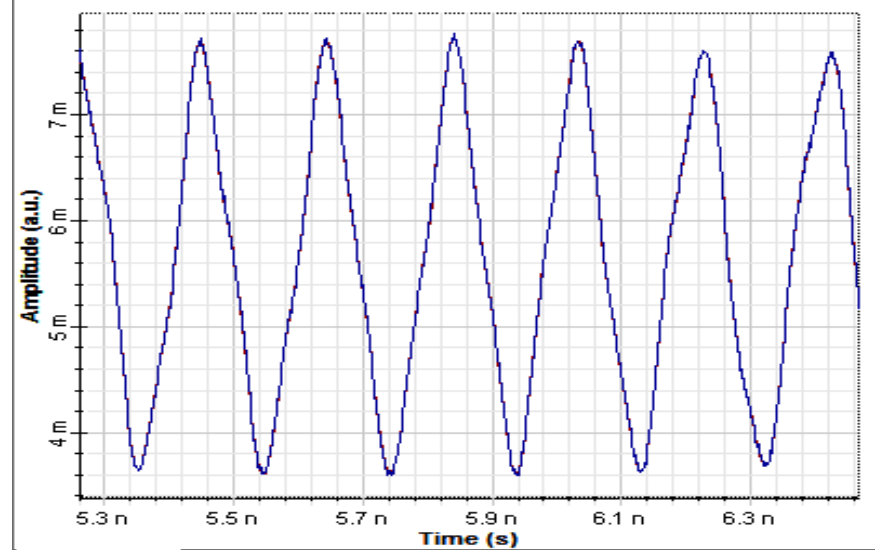

Fig. 5. The generated triangular shaped waveform

\section{Conclusion}

In this approach, by reducing system complexity and cost by avoiding frequency converter and reducing the consumption of fiber, we produce triangular-shaped microwave pulses. By the amplification effect of SBS gain, the suppressed optical carrier is retrieved back and by means of an optical filter, all optical sidebands with negative-orders are eliminated then. The first order harmonics amplitude is often tuned to 9 fold of that of third order harmonics by opto-electronic conversion in photo detector. The modulated signal processing using SBS is then carried out. And it must be noted that when the SBS gain is at peak, 
nonlinear phase change induced by SBS is zero, which is a crucial provision to our method. Consequently, an OBPF filtered out the unnecessary optical sidebands, except the positive first order sidebands, the positive third order sidebands and the carrier. The second order harmonics are often ignored during the optical to electrical conversion in a photo detector, as the strength of optical carrier is far greater than that of the positive first order optical sideband. And to contribute gain for retrieving the suppressed carrier, the carrier optical processing based on stimulated Brillouin scattering is implemented. For eliminating the unnecessary sidebands, an optical band pass filter is then employed. The triangular shaped microwave pulses are often produced in this manner as the SBS gain to be sufficiently huge. The target triangular shaped microwave pulse was produced with the efficiency and therefore the tunability of the suggested system was confirmed. The Triangular Shaped microwave pulse always has its uniqueness in the Communication Systems. The generation can be considerably simplified and complexity can also be reduced in the future. And therefore the other non-linearities present in the fiber are often used as advantages in generating different types of waveforms. Tunability of the waveform also can be improvised in the future generation techniques.

\section{References}

[1] Wei Li, Ning Hua Zhu, Li Xian Wang, W. Li et al. "Harmonic RF carrier generation and broadband data upconversion using stimulated Brillouin scattering", Optics Communications, 3437-3439, Elsevier 2011.

[2] Michael Sauer, Andrey Kobyakov, A. Boh Ruffin "Radio-Over-Fiber Transmission with Mitigated Stimulated Brillouin Scattering”, IEEE photonics technology letters, October 1, 2007; vol. 19, no. 19.

[3] Y. G. Shee, M. H. Al-Mansoori, S. Yaakob, A. Man, A. K. Zamzuri, F. R. Mahamd, Adikan and M. A. Mahdi, "Millimeter wave carrier generation based on a double-Brillouin-frequency spaced fiber laser", Optical Society of America, 2012.

[4] Lute Maleki and Andrey B.Matsko "Optical Generation of Microwave Reference Frequencies", OEwaves Inc., 2555 East Colorado Blvd., Pasadena, California 91107, USA.

[5] N. M. Nawawi, S. M. Idrus, "Investigation of Stimulated Brillouin Scattering for the Generation of Millimeter Waves for Radio over Fiber System", IEEE 2008 2nd Malaysia Conference on Photonics, 26-27 August 2008, Putrajya, Malaysia.

[6] Shiming Gao, Ying Gao, Hongyan Fu, "Fiber Stimulated Brillouin Scattering-Based Microwave Signal Processing", Proc. of SPIE, 2008; Vol. 7134 71341V-1.

[7] A. García-Juárez*a, I. E. Zaldívar-Huertab, G. Aguayo-Rodríguezb, J. Rodríguez-Asomozac, R, "Optical heterodyne technique for generating and distributing microwave signals", Proc. of SPIE, 2010; Vol. 7620 762009-1. 